ARTICLE

DOI: $10.1038 / s 41467-018-04937-8$

\title{
High energy flexible supercapacitors formed via bottom-up infilling of gel electrolytes into thick porous electrodes
}

Xiangming $\mathrm{Li}^{1,2}$, Jinyou Shao ${ }^{1}$, Sung-Kon Kim²,3, Chaochao Yao ${ }^{1}$, Junjie Wang $\mathbb{0}^{2}$, Yu-Run Miao ${ }^{3}$, Qiye Zheng ${ }^{2}$, Pengcheng Sun ${ }^{2}$, Runyu Zhang ${ }^{2} \&$ Paul V. Braun (i) ${ }^{2,4}$

Formation of thick, high energy density, flexible solid supercapacitors is challenging because of difficulties infilling gel electrolytes into porous electrodes. Incomplete infilling results in a low capacitance and poor mechanical properties. Here we report a bottom-up infilling method to overcome these challenges. Electrodes up to $500 \mu \mathrm{m}$ thick, formed from multi-walled carbon nanotubes and a composite of poly(3,4-ethylenedioxythiophene), polystyrene sulfonate and multi-walled carbon nanotubes are successfully infilled with a polyvinyl alcohol/ phosphoric acid gel electrolyte. The exceptional mechanical properties of the multi-walled carbon nanotube-based electrode enable it to be rolled into a radius of curvature as small as $0.5 \mathrm{~mm}$ without cracking and retain $95 \%$ of its initial capacitance after 5000 bending cycles. The areal capacitance of our $500 \mu \mathrm{m}$ thick poly(3,4-ethylenedioxythiophene), polystyrene sulfonate, multi-walled carbon nanotube-based flexible solid supercapacitor is $2662 \mathrm{mF} \mathrm{cm}^{-2}$ at $2 \mathrm{mV} \mathrm{s}^{-1}$, at least five times greater than current flexible supercapacitors.

\footnotetext{
${ }^{1}$ Micro-/Nano-technology Research Center, State Key Laboratory for Manufacturing Systems Engineering, Xi'an Jiaotong University, 710049 Xi'an, Shaanxi, China. ${ }^{2}$ Department of Materials Science and Engineering, Frederick Seitz Materials Research Laboratory, and Beckman Institute for Advanced Science and Technology, University of Illinois at Urbana-Champaign, Urbana, IL 61801, USA. ${ }^{3}$ School of Chemical Engineering, Chonbuk National University, 567 Baekjedaero, Deokjin-gu, Jeonju-si, Jeollabuk-do 54896, Republic of Korea. ${ }^{4}$ Department of Chemistry, University of Illinois at Urbana-Champaign, Urbana, IL

61802, USA. Correspondence and requests for materials should be addressed to J.S. (email: jyshao@xitu.edu.cn) or to P.V.B. (email: pbraun@illinois.edu)
} 
E lexible solid supercapacitors (FSSCs) are usually constructed by sandwiching a gel electrolyte between a pair of porous electrodes. During this process, the gel electrolyte partially infills the pores of the electrodes forming the electrode-electrolyte interface $^{1-3}$. Such designs have drawn attention because of their mechanical flexibility, relatively light weight, potential for low cost, and minimal issues with electrolyte leakage, with the target application typically flexible electronics ${ }^{1-7}$. The FSSC community has primarily focused on advancing the electrode and electrolyte materials in the past decades ${ }^{8-18}$, with only minimal attention paid to developing effective strategies for maximizing formation of the electrode-electrolyte interface (the part of the structure that stores energy). Formation of this interface is the precondition for a high energy density, but remains a considerable obstacle for FSSCs. Current state-of-the-art FSSCs utilize thin electrodes as thin electrodes provide mechanical flexibility and because the required solid electrolyte can infill a thin porous electrode to form a good high area electrode-electrolyte interface. However, thin electrodes, typically ranging from $10 \mathrm{~s}$ of nm to a few $10 \mathrm{~s}$ of $\mu \mathrm{m}$ in thickness, tend to have low areal capacitances since capacitance scales with electrode volume $e^{8-23}$. For example, while a $500 \mathrm{~nm}$ thick electrode formed from a multi-layer graphene/carbon nanotube structure exhibited a volumetric capacitance of $49.5 \mathrm{~F} \mathrm{~cm}^{-3}$, the areal capacitance was only $2.54 \mathrm{mF} \mathrm{cm}^{-2}{ }^{14}$. A $100 \mathrm{~nm}$ thick nano-porous gold/polypyrrole electrode exhibited a volumetric capacitance of $150 \mathrm{~F} \mathrm{~cm}^{-3}$, yet its areal capacitance was only $1.8 \mathrm{mF} \mathrm{cm}^{-2} 11$. An ultrathin (25 nm thick) graphene electrode had a gravimetric capacitance of $285 \mathrm{~F} \mathrm{~g}^{-1}$, yet the areal capacitance was just $0.45 \mathrm{mF} \mathrm{cm} \mathrm{cm}^{-2}$.

The obvious solution to increase the areal capacitance is to thicken the electrodes ${ }^{23}$. However, the areal capacitance of reported FSSCs did not scale with electrode thickness, usually saturating while the electrode was quite thin. For example, the areal capacitance of a FSSC based on single wall carbon nanotubes saturates at an electrode thickness of $2 \sim 3 \mu \mathrm{m}$, probably because the gel electrolyte could only infill the electrode up to this thickness ${ }^{19,24}$. Prior to the report here, the only route to improve the infilling of the electrode was to add macropores to the structure. Examples of FSSC containing both micropores and larger macropores include one formed using $120 \mu \mathrm{m}$ thick 3D graphene hydrogel electrodes which exhibited an areal capacitance of $372 \mathrm{mF} \mathrm{cm}^{-2} 24$, one formed with $81.6 \mu \mathrm{m}$ thick reduced graphene oxide/polypyrrole nanotube paper electrodes which exhibited an areal capacitance of $512 \mathrm{mF} \mathrm{cm}^{-2} 25$, and one formed using graphene/carbon nanofiber aerogel electrodes which showed an areal capacitance of $158 \mathrm{mF} \mathrm{cm}^{-2} 26$. In these systems, the macropores both serve as an electrolyte reservoir and improve the infilling of the porous electrodes ${ }^{24-28}$. However, the macropores decreased the overall electrode surface area, decreasing the areal capacitance. To maximize capacitance, an electrode should be thick (e.g., $500 \mu \mathrm{m}$ ), have a minimum of macropores, and maximum of micropores or mesopores. However, prior to the work here, it has not been possible to infill such a structure with solid electrolyte.

Thick pseudocapacitive electrodes based on 3D-structured transition metal oxides provide much higher areal capacitances ${ }^{29-}$ 31. The areal capacitance of a $\mathrm{MnO}_{2}$-coated $\sim 270 \mu \mathrm{m}$ thick $3 \mathrm{D}$ silicon scaffold reached $670 \mathrm{mF} \mathrm{cm}{ }^{-2}$ at $2 \mathrm{mV} \mathrm{s}^{-1}$ using a $0.5 \mathrm{M}$ $\mathrm{Na}_{2} \mathrm{SO}_{4}$ aqueous electrolyte ${ }^{29}$, the areal capacitance of a microsupercapacitor formed using a $\sim 15 \mu \mathrm{m}$ thick 3D laser-scribed graphene- $\mathrm{MnO}_{2}$ electrode reached $852 \mathrm{mF} \mathrm{cm}{ }^{-2}$ using a $1 \mathrm{M}$ $\mathrm{Na}_{2} \mathrm{SO}_{4}$ aqueous electrolyte ${ }^{30}$, and the areal capacitance for $3 \mathrm{D}$ structured $25 \mu \mathrm{m}$ thick $\mathrm{Au} / \mathrm{RuO}_{2} \cdot x \mathrm{H}_{2} \mathrm{O}$ reached $3473 \mathrm{mF} \mathrm{cm}{ }^{-2}$ at $0.1 \mathrm{mV} \mathrm{s}^{-1}$ in a $0.5 \mathrm{M} \mathrm{H}_{2} \mathrm{SO}_{4}$ electrolyte ${ }^{31}$. While these high areal capacitances would be highly desirable for FSSCs, the brittle nature of metal oxides, coupled with the fact that liquid electrolytes are commonly used for pseudocapacitors, makes building a FSSC using these electrodes challenging.

Starting from our understanding of why it is difficult to infill thick porous electrodes we create a new bottom-up method to infill gel electrolytes into porous electrodes as thick as $500 \mu \mathrm{m}$. Using this method, high capacity thick electrodes are infilled with gel electrolytes, and FSSCs are formed from these electrodes. The highest capacity FSSC is realized using bottom-up infilled composite electrodes containing a poly(3,4-ethylenedioxythiophene), polystyrene sulfonate (PEDOT/PSS) blend and multi-walled carbon nanotubes (MWCNT). The FSSC formed using this electrode exhibits an areal capacitance of 2662 $\mathrm{mF} \mathrm{cm} \mathrm{cm}^{-2}$, about five times greater than current state-of-the-art FSSCs.

\section{Results}

Top-down vs. bottom-up infilling method. Figure 1 illustrates why it is difficult to infill a gel electrolyte into thick porous electrodes, and our suggested solution. As illustrated in Fig. 1a and Supplementary Figure 1, during the top-down gelation process the volume shrinkage, and the dramatic increase in viscosity as the gel point is approached, leads to incomplete infilling. As water evaporates from the electrolyte (a mixture of water, poly (vinyl alcohol) (PVA) and $\mathrm{H}_{3} \mathrm{PO}_{4}$ ), a gel layer is generated at the air-electrolyte interface (the first two snapshots in Supplementary Figure 1), which thickens as the water continues to evaporate. As shown by the last two snapshots in Supplementary Figure 1, the gel is sufficiently solid that it can be mechanically removed from the top of the electrolyte solution. Because the gel forms from the top-down, it is very difficult to infill a thick porous electrode via the commonly used top-down infilling process (i.e., via drop casting) (Fig. 1a). As the water evaporates, a viscous skin forms above the electrode as illustrated in Fig. 1a. Because the volume of PVA and $\mathrm{H}_{3} \mathrm{PO}_{4}$ in the liquid below the skin starts out as typically $<10 \%{ }^{32}$, once this liquid dries in the gel, only a small proportion of the electrode pore volume is occupied by the gel and most of the pore space is empty. As the pore sizes in the electrode become smaller, the infilling problem only becomes worse, because viscous resistance to flow grows ${ }^{33,34}$.

In the bottom-up infilling method, the electrolyte solution is directly cast onto a porous electrode located on a gas-permeable substrate (Fig. 1b). A water impermeable film (e.g., polyester $(\mathrm{PET}))$ is then placed over the electrolyte solution, forcing the water in electrolyte solution to evaporate downwards through the gas-permeable substrate. The downward evaporation of water starts the formation of the gel electrolyte from the bottom of the porous electrode; the gel then grows upwards into the electrode. No voids form in the pores of the electrode because capillary forces drive the liquid solution to continuously refill the pores, as indicated by the yellow arrows in the second scheme in Fig. 1b. Since the capillary force is inversely proportional to the pore size, as an added benefit of this infilling approach, we suspect smaller pore electrodes may infill better than larger pore electrodes. After removing the impermeable cover and the gas-permeable substrate, a flexible electrode with a well-infilled gel electrolyte is obtained.

Electrode infilling. Because of their high surface area, stability, mechanical flexibility, and electrical conductivity, carbon nanotube-based electrodes are commonly used for FSSC ${ }^{2,4,35-37}$. However, these electrodes usually have small and complex pores which are difficult to infill with a gel electrolyte. For the study here, we specifically use a thick (typically $\sim 500 \mu \mathrm{m}$ ) electrode formed from randomly distributed multi-walled carbon nanotubes (MWCNTs, $\sim 50 \mathrm{~nm}$ in diameter). Figure 1c shows scanning 
a
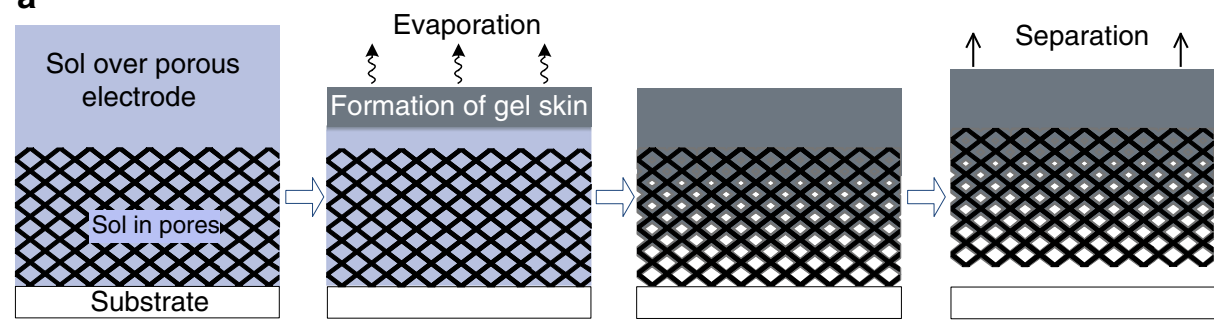

b

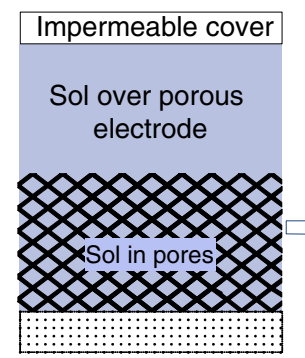

Gas-permeable substrate
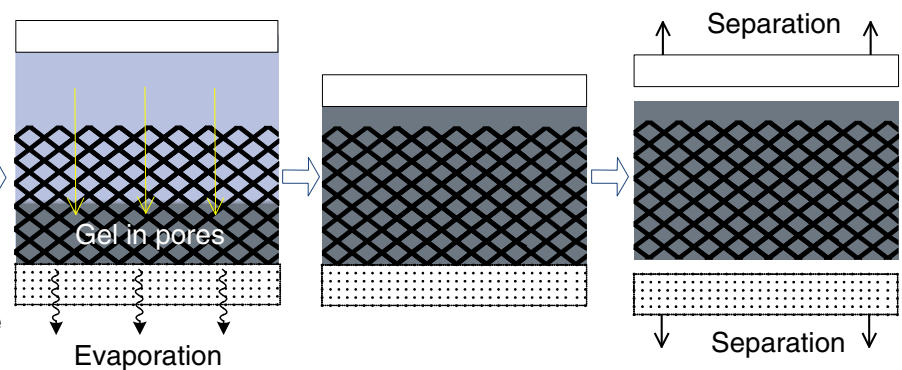

C
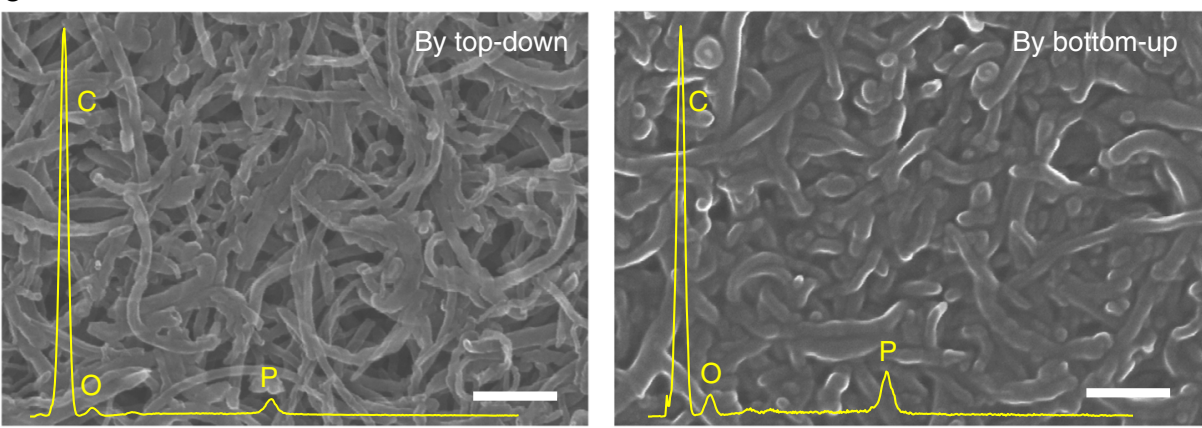

Fig. 1 Top-down vs bottom-up infilling. a The top-down infilling method. Left to right: sol is casted onto a porous electrode; sol above the porous electrode gels forming a skin; sol in the porous electrode gels, leaving behind voids; a free-standing incompletely filled electrode is obtained after the separation from the substrate. $\mathbf{b}$ The bottom-up infilling method. Left to right: sol is cast on a porous electrode located on a gas-permeable substrate and covered with an impermeable film; the gel forms from the bottom-up until the entire porous electrode is infilled with gel; a free-standing gel-filled electrode is obtained after removal of the substrate. c SEM images of the top of the MWCNT electrodes infilled with gel electrolyte by the top-down (left) and bottom-up (right) methods. Overlaid on the images are energy dispersive spectroscopy scans where the phosphorus peaks indicate the presence of the electrolyte. Scale bar for $\mathbf{c}$ is $200 \mathrm{~nm}$

electron micrograph (SEM) images of MWCNT electrodes infilled with gel electrolyte by the top-down and bottom-up methods. Using the bottom-up infilling method, the gel electrolyte infills the entire MWCNT electrode and minimal voiding is observed. Using the top-down infilling method, the amount of gel electrolyte in the porous electrode decreases significantly from the bottom to the top of the electrode (Supplementary Figure 2). Phosphorus mapping via energy dispersive spectroscopy (EDS) also suggests a different degree of gel infilling (the gel contains $\mathrm{H}_{3} \mathrm{PO}_{4}$ ); compare the intensity of the P peak in the spectrums in Fig. 1c. Using the bottom-up method, the atomic ratio between carbon and phosphorus is nearly constant at 1:0.051 across the cross section of the MWCNT electrode, indicating a uniform distribution of gel electrolyte. Using the top-down infilling method, the value decreases from 1:0.05 at the bottom of the electrode to 1:0.014 at the top of the electrode, suggesting a significant decline of gel infilling from bottom to the top of the porous electrodes. The degree of gel infilling of the electrode pores can be quantified using Brunauer-Emmett-Teller surface area analysis and Barrett-Joyner-Halenda pore size and volume analysis (Supplementary Figure 3). The pore volume of the electrode infilled using the bottom-up method is half than that infilled using the top-down method, and the pore sizes are also significantly decreased for the bottom-up infilled electrode. The difference in the gel infilling of the electrode by the top-down and bottom-up methods is quantitatively determined via a simple test using a micrometer (Supplementary Figure 4).

FSSC electrical properties. Figure 2 shows the electrical properties of FSSCs formed via top-down and bottom-up infilling of 500$\mu \mathrm{m}$-thick MWCNT electrodes. Figure 2a, b and Supplementary Figure 5 show two-electrode cyclic voltammetry (CV) and galvanostatic charge-discharge (GCD) curves for FSSC formed using the two-electrode preparation methods. The FSSC fabricated by the top-down method shows a much smaller capacitance than one formed by the bottom-up method. There are several reasons for this. First, the top-down infilled electrode is incompletely filled, and thus part of the structure is not contributing to the capacitance. Second, in the partially filled regions, the ions have to bypass the voids during charging and discharging, leading to long diffusion paths, and consequently an increased equivalent series 

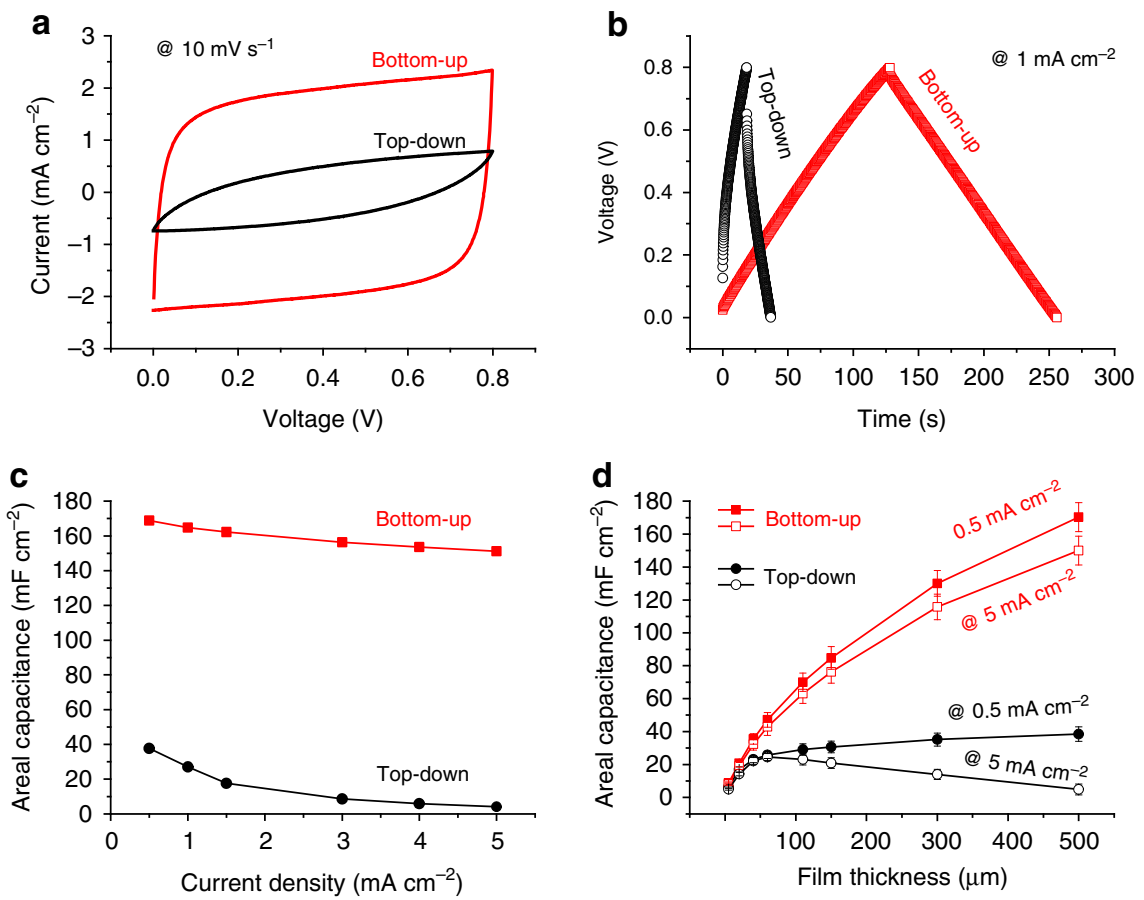

Fig. 2 Electrochemical properties of the top-down and bottom-up infilled FSSCs. a CV and $\mathbf{b}$ GCD curves of the different FSSCs. c Areal capacitances of the top-down and bottom-up infilled FSSCs at different current densities. $\mathbf{d}$ Thickness dependence of the areal capacitance for top-down and bottom-up infilled FSSC devices at 0.5 and $5 \mathrm{~mA} \mathrm{~cm}^{-2}$

resistance (ESR) which tends to result in a lower power and energy capability $^{38}$. The ESR for the bottom-up $\left(9.3 \Omega \mathrm{cm}^{2}\right)$ and topdown $\left(96 \Omega \mathrm{cm}^{2}\right)$ infilled FSSC can be obtained from the Nyquist plots (Supplementary Figure 6) ${ }^{39}$.

As shown in Fig. 2c, the areal capacitance and rate performance of the bottom-up infilled FSSC is significantly greater than the top-down infilled FSSC. The areal capacitance of the bottom-up infilled FSSC is ca. $164.8 \mathrm{mF} \mathrm{cm}^{-2}$ at a current density of $1 \mathrm{~mA} \mathrm{~cm}^{-2}$, which is six times the $27 \mathrm{mF} \mathrm{cm} \mathrm{cm}^{-2}$ exhibited by the FSSC formed by top-down infilling at the same current density. Clearly, increasing the degree of infilling of the gel electrolyte in the thick porous electrode increases the accessible surface area of the electrode and thus the overall capacity ${ }^{4041}$. Along with an improvement in capacity, the rate performance (Fig. 2c) is also significantly improved by the bottom-up infilling method as estimated from the GCD profiles at current densities ranging from 0.5 to $5 \mathrm{~mA} \mathrm{~cm}^{-2}$. The areal capacitance for the bottom-up infilled FSSC is ca. $168.8 \mathrm{mF} \mathrm{cm}^{-2}$, at a discharge current $0.5 \mathrm{~mA} \mathrm{~cm}^{-2}$, which is nearly five times the capacitance of the FSSC formed by the top-down infilling method at the same current density. The capacitance of bottom-up infilled FSSC remains as high as $151.2 \mathrm{mF} \mathrm{cm}^{-2}$ at the high discharge current of $5 \mathrm{~mA} \mathrm{~cm}^{-2}$ ( $89 \%$ capacity retention relative to the 0.5 $\mathrm{mA} \mathrm{cm}{ }^{-2}$ discharge). In contrast, the top-down infilled FSSC retains only $10 \%$ of its capacitance $\left(4.1 \mathrm{mF} \mathrm{cm}^{-2}\right)$ under the same test conditions. This difference in rate ability is also apparent in the frequency-dependent impedance measurements (Supplementary Figure 6).

The bottom-up method enables scaling of the areal capacitance with electrode thickness. Figure $2 \mathrm{~d}$ shows the dependence of the areal capacitance with thickness as a function of current density and infilling method. For the top-down method, the areal capacitance saturates with increasing electrode thickness at a current density of $0.5 \mathrm{~mA} \mathrm{~cm}{ }^{-2}$, similar to other literature reports ${ }^{19,24,42}$. At the higher current density of $5 \mathrm{~mA} \mathrm{~cm}^{-2}$, the capacitance even starts to decline once the electrode is thicker than about $50 \mu \mathrm{m}$ (Fig. 2d), probably due to kinetic limitations caused by voids in the electrode. In contrast, using the bottom-up infilling method, the areal capacitance increases with electrode thickness until at least $500 \mu \mathrm{m}$. It should be noted that the areal capacitance does not linearly increase with the electrode thickness, in particular at high current density, because the mean ion diffusion length increases with electrode thickness. Since the volume of the MWCNT electrode per square centimeter is directly proportional to the thickness, the volumetric capacitance of FSSCs shows similar trends as the thickness dependence (Supplementary Figure 7). At $10 \mathrm{~mA} \mathrm{~cm}^{-3}$ for example, the volumetric capacitance for the top-down infilled FSSC, on an electrode volume basis decreases from $6.1 \mathrm{~F} \mathrm{~cm}^{-3}$ for the $5 \mu \mathrm{m}$ thick electrode to $0.39 \mathrm{~F} \mathrm{~cm}^{-3}$ for the $500 \mu \mathrm{m}$ thick electrode, while, the volumetric capacitance for the bottom-up infilled FSSC decreases significantly less, from 6.37 to $1.71 \mathrm{~F} \mathrm{~cm}^{-3}$. We find the volumetric capacitance of even the thick $(>100 \mu \mathrm{m})$ bottom-up infilled FSSC is comparable to the value of other previously reported, much thinner, sandwich type MWCNT-based thin-film FSSCs $^{43-45}$.

The electrochemical performances of FSSCs infilled with the gel electrolyte were additionally compared over a voltage window of 0 to $1 \mathrm{~V}$ with the same electrode filled with $1 \mathrm{M} \mathrm{H}_{2} \mathrm{SO}_{4}$ aqueous electrolyte (Supplementary Figure 8). As anticipated, the capacitance, rate performance and ESR of the liquid electrolyte filled electrode was better than for the electrodes infilled with the gel electrolyte because of the better ion conductivity of the aqueous electrolyte than the gel electrolyte. However, liquid electrolytes lead to serious packaging challenges for flexible systems.

Mechanical performance. For practical application of FSSCs, mechanical performance is important ${ }^{1-3,9}$. We find the improved infilling of gel electrolyte in the porous electrode by the bottomup method significantly improves mechanical flexibility and 
a

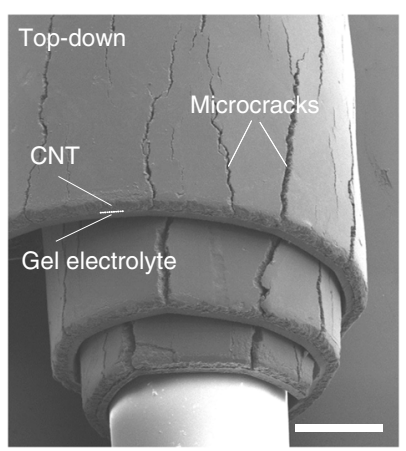

b

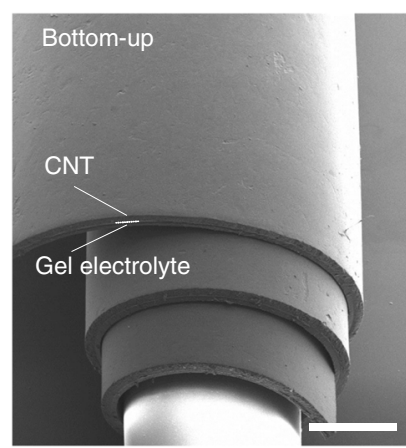

C

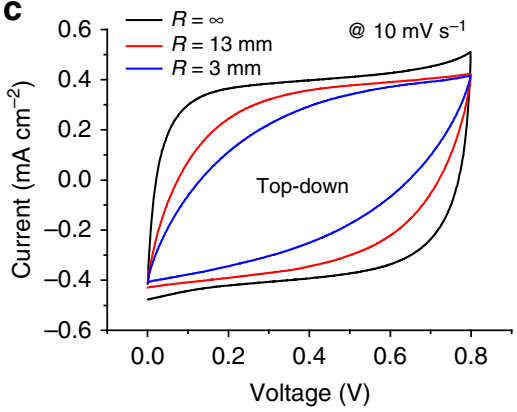

d

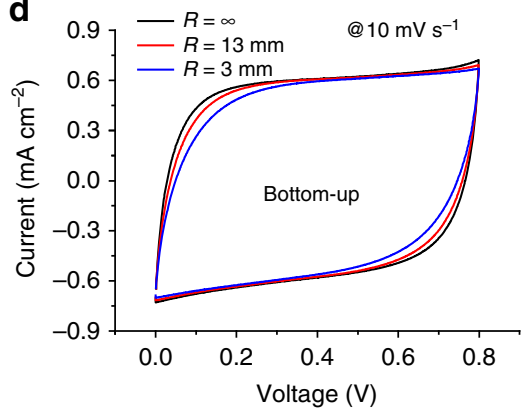

e

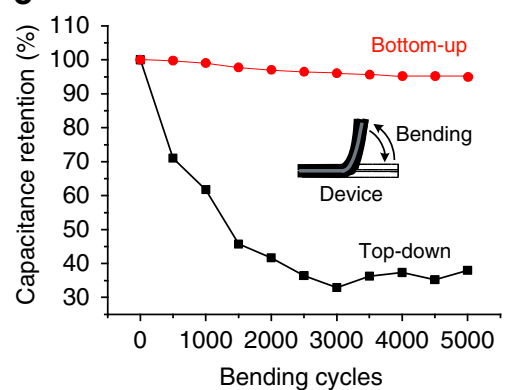

Fig. 3 Gel filling method dependent mechanical stability. a, b The gel infilling-dependent microcracks (or lack of microcracks) in $150 \mu \mathrm{m}$ in thick MWCNT electrodes rolled-up over a glass tube with a radius of $\sim 0.5 \mathrm{~mm}$ fabricated by the two methods. Electrochemical performance of $\mathbf{c}$ and $\mathbf{d}$ once rolled-up FSSC devices and e after repeated bending. In e the capacitance retention with the bending number is confirmed by GCD at a current density of 0.15 mA after every 500 bends at bending radius of curvature of $5.7 \mathrm{~mm}$. Scale bar for $\mathbf{a}$ and $\mathbf{b}$ is $500 \mu \mathrm{m}$

stability (Fig. 3). The $\mathrm{PVA} / \mathrm{H}_{3} \mathrm{PO}_{4}$ gel polymer electrolyte is flexible and elastic at room temperature, and serves to bind the electrode together ${ }^{46}$, which improves the mechanical properties of the porous electrode. As shown in Fig. 3a, b, the top-down infilled electrodes exhibit microcracks with bending, while the bottom-up infilled electrode exhibits no cracking even the electrode is rolled-up over a glass tube with a radius as small as 0.5 $\mathrm{mm}$. Even if they do not lead to complete failure, microcracks are problematic as they will increase the ESR and may even electrically isolate parts of the electrode, which will harm the device electrochemical performance. Figure $3 \mathrm{c}$, d compare CV curves of rolled-up FSSC devices formed from $\sim 150 \mu \mathrm{m}$ thick electrodes infilled by the two different methods. For a FSSC device formed by the top-down method, the cathodic/anodic current values decrease with a decreasing rolled-up radius, which we ascribe to electrode cracking. The CV curves also become significantly distorted as the rolled-up radius decreases, indicative of a crackinduced large ESR ${ }^{47}$. The ESR values increased from $28.7 \Omega \mathrm{cm}^{2}$ before bending, to $108.8 \Omega \mathrm{cm}^{2}$ for a bending radius, $R$, of $13 \mathrm{~mm}$, to $252.6 \Omega \mathrm{cm}^{2}$ for $R=3 \mathrm{~mm}$, as calculated from the IR drops on GCD plots at the corresponding radii (Supplementary Figure 9). The bottom-up infilled structures exhibit considerable stability to mechanical cycling (Fig. 3e). The capacitance retention of the thick FSSC with the well-infilled gel electrolyte is above $95 \%$ after 5000 bending cycles with a radius curvature of $5.7 \mathrm{~mm}$, while the capacitance retention is only $40 \%$ for the FSSC with less infilled gel electrolyte. In contrast to the top-down infilled structures, CV curves of the bottom-up infilled rolled-up FSSC remain almost unchanged as a function of rolled-up radii (Fig. 3d), demonstrating the electrode stability under different bending stresses. The difference in capacity of the two-electrode designs is almost certainly due to microcracks in the less infilled electrodes (Fig. 3a and Supplementary Figure 10), which increases the ESR from 28.7 $\Omega \mathrm{cm}^{2}$ before the first bending cycle to $107 \Omega \mathrm{cm}^{2}$ after 5000 bending cycles (Supplementary Figure 11).
Practical considerations. For practical application, the FSSC electrochemical stability, leakage current and self-discharge rate are important. The thick bottom-up infilled FSSCs show good performance in all these areas. After 10,000 GCD cycles at a constant current of $10 \mathrm{~mA} \mathrm{~cm}^{-2}$, a $150 \mu \mathrm{m}$ thick bottom-up infilled FSSC retains $98 \%$ of initial capacitance and a columbic efficiency near unity, indicating a good cycle life (Supplementary Figure 12). The leakage current was measured to be $2.5 \mu \mathrm{A}$ after $12 \mathrm{~h}$ (Supplementary Figure 13), which is comparable to commercial supercapacitors ${ }^{40}$. Specifically, the time required for the voltage across the supercapacitor to decrease from $V_{\max }$ (i.e., $1 \mathrm{~V}$ ) to $1 / 2 V_{\max }$ is $>24 \mathrm{~h}$ (Supplementary Figure 13) compared to 8-21 $\mathrm{h}$ for commercial supercapacitors ${ }^{48}$.

Tandem devices. Tandem bottom-up infilled FSSCs are configured in series and in parallel to increase the voltage window or capacitive current, respectively (Supplementary Figure 14). The tandem serial FSSCs are operated over a $1.6 \mathrm{~V}$ window (twice that of a single FSSC), and as expected, there is a reduction in the current because of the increased series resistance, as confirmed by CV and GCD (Supplementary Figure 14). The tandem parallel FSSCs provided twice the current of a single FSSC, while maintaining a steady capacitive behavior throughout the $0.8 \mathrm{~V}$ voltage window. After charging at $3 \mathrm{~V}$ for $24 \mathrm{~min}$, a device of three FSSCs linked in series can light a blue LED for $\sim 10$ min (the LED turnon voltage is $\sim 2 \mathrm{~V}$, thus the requirement of several FSSCs in series) (Supplementary Figure 15a). Owing to the mechanical stability of the bottom-up infilled FSSCs, the device of three FSSCs linked in series can be rolled around a cylindrical pencil (radius of $\sim 4 \mathrm{~mm}$ ) while still powering the LED (Supplementary Figure 15b).

Bottom-up infilled pseudocapacitor. To demonstrate the bottom-up infilling versatility, a composite $70 \mathrm{wt} \%$ PEDOT/PSS 

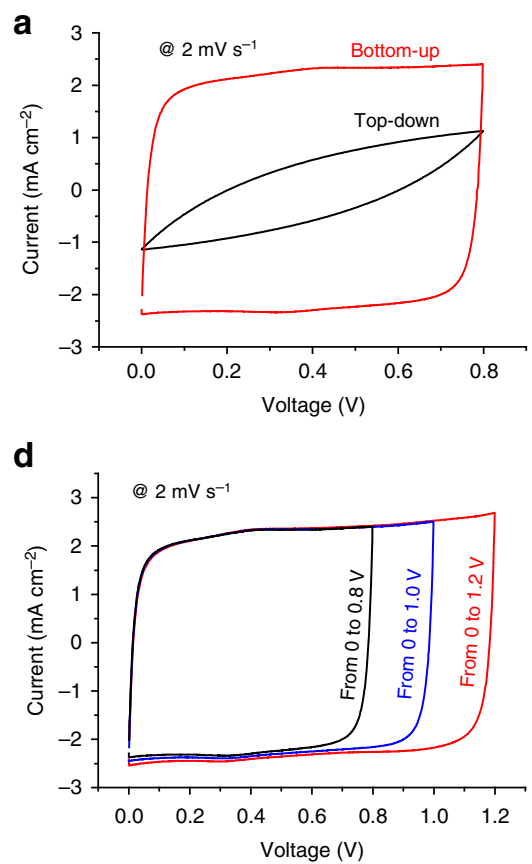
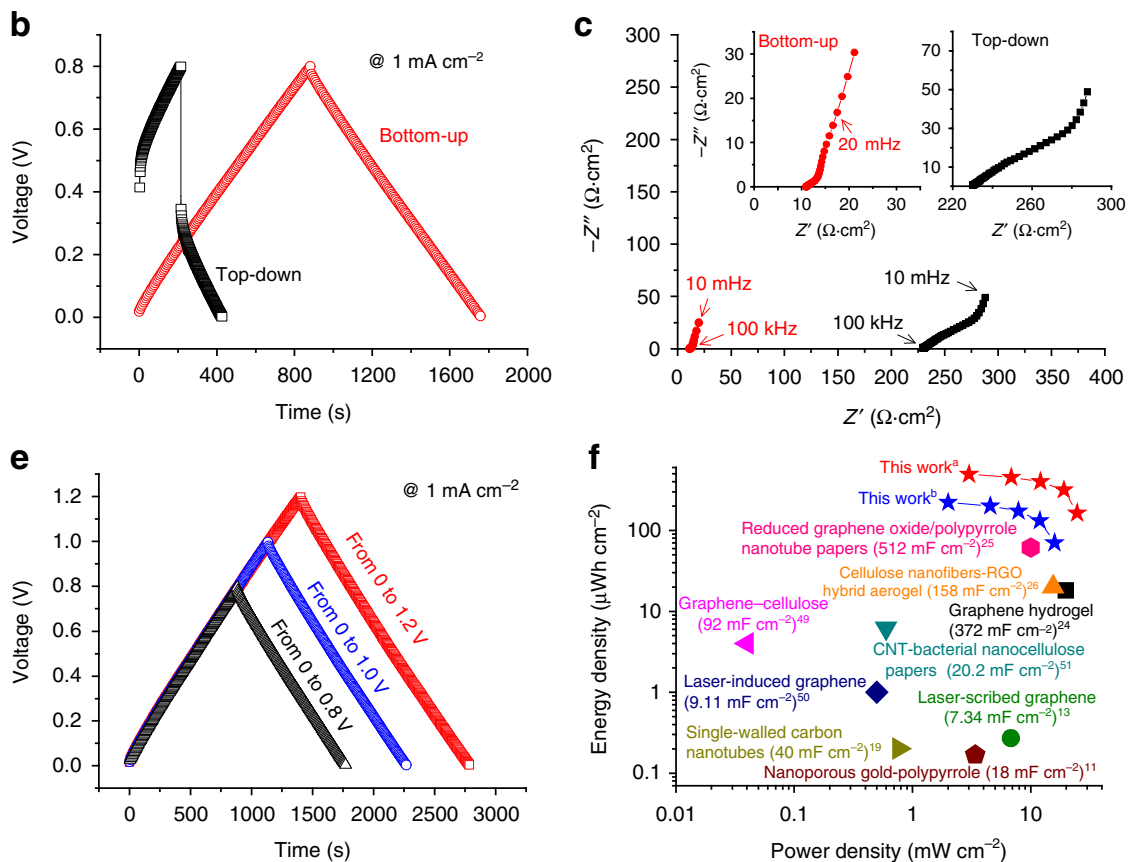

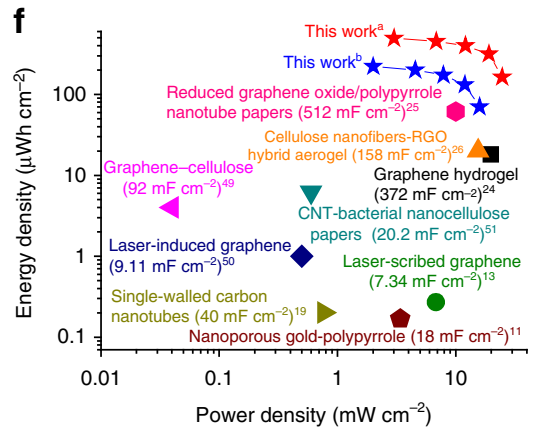

Fig. 4 Electrical performance of FSSCs formed using $500 \mu \mathrm{m}$ thick PEDOT/PSS-WMCNT electrodes. a CV, b GCD profiles, and $\mathbf{c}$ Nyquist plots (100 kHz to $10 \mathrm{mHz}$ ) of the top-down and bottom-up designs. Note that the inset is the plot for the bottom-up infilled FSSC. d CV and e GCD curves over different potential windows for a FSSC formed from bottom-up infilled $500 \mu \mathrm{m}$ thick PEDOT/PSS-MWCNT electrodes. $\mathbf{f}$ Areal Ragone plot (per $\mathrm{cm}^{2}$ of the FSSC device). This work ${ }^{\mathrm{a}}$ and this work ${ }^{\mathrm{b}}$ refers to the bottom-up infilled FSSCs with an electrode thicknesses of $500 \mu \mathrm{m}$ tested over potential windows of 0 to 1.2 $\mathrm{V}$ and 0 to $0.8 \mathrm{~V}$, respectively

$30 \mathrm{wt} \%$ MWCNT electrode (Supplementary Figure 16), is used for fabricating FSSCs. In this design, the conductive polymer, PEDOT/PSS, enhances the mechanical properties by serving as a binder for the MWCNTs, and the capacitance is increased due to the pseudocapacitive properties of PEDOT. The composite electrode has good mechanical flexibility (Supplementary Figure 17), and perhaps more importantly, a considerable mechanical strength. The yield stress is at least $7 \mathrm{MPa}$ (Supplementary Figure 17), which should make it attractive for FSSCs. The electrochemical performances of FSSCs formed using $500-\mu \mathrm{m}$-thick topdown and bottom-up infilled PEDOT/PSS-MWCNT electrodes are compared (Fig. 4). The bottom-up infilled FSSC based on 500$\mu \mathrm{m}$-thick PEDOT/PSS-MWCNT electrodes exhibits rectangularshaped CV curves (Fig. 4a, Supplementary Figure 18a) and symmetric triangular-shaped GCD profiles (Fig. 4b, Supplementary Figure 18b), while the top-down infilled FSSC provides distorted CV curves and asymmetric GCD profiles at the same voltage scan rate and current density. The differences are particularly obvious in the Nyquist plots (Fig. 4c). The ESR for bottom-up infilled FSSC is $11.2 \Omega \mathrm{cm}^{2}$, while for the top-down infilled FSSC it is $231.3 \Omega \mathrm{cm}^{2}$.

The areal capacitance of the bottom-up infilled FSSC cell formed from the $500 \mu \mathrm{m}$ thick electrodes is $2662 \mathrm{mF} \mathrm{cm}^{-2}$ at 2 $\mathrm{mV} \mathrm{s}^{-1}$ (Fig. 4a), at least five times greater than the previously reported state-of-the-art $\left(500 \mathrm{mF} \mathrm{cm} \mathrm{cm}^{-2} \text { or less }\right)^{9-19,24-26,49-51}$. The $500 \mu \mathrm{m}$ thick bottom-up infilled FSSC retains an areal capacitance of $2038 \mathrm{mF} \mathrm{cm}{ }^{-2}$ at a scan rate of $10 \mathrm{mV} \mathrm{s}^{-1}$ (Supplementary Figure 18). In comparison, the areal capacitance of the $500 \mu \mathrm{m}$ thick top-down infilled FSSC decreased from 582.6 $\mathrm{mF} \mathrm{cm}{ }^{-2}$ at $2 \mathrm{mV} \mathrm{s}^{-1}$ to $55.7 \mathrm{mF} \mathrm{cm}^{-2}$ at $10 \mathrm{mV} \mathrm{s}^{-1}$ (Supplementary Figure 18). It is also notable how much greater the areal capacitance of the bottom-up infilled PEDOT/PSS-MWCNTbased FSSC is than one formed using only MWCNT (compare Fig. 2a, b with Fig. $4 a, b)$. This difference is perhaps due to the presence of the electrically conductive PEDOT, and probably more importantly the pseudocapacitance of PEDOT ${ }^{52,53}$. The loading of PEDOT/PSS in the composite electrodes influences the capacitance of FSSCs and we found the optimal value to be $\sim 70$ wt.\% in our experiments (Supplementary Figure 19). The bottomup infilled PEDOT/PSS-MWCNT-based FSSC also provides a good rate performance relative to a top-down infilled PEDOT/ PSS-MWCNT electrode (Supplementary Figure 20a) and provides a capacitance retention of $>93 \%$ after 10,000 charging/ discharging cycles at a current density of $15 \mathrm{~mA} \mathrm{~cm}^{-2}$ (Supplementary Figure 20b).

When the cell voltage increased from 0.8 to $1.2 \mathrm{~V}$, CV curves of FSSC formed using bottom-up infilled 500- $\mu$ m-thick PEDOT/ PSS-MWCNT remained rectangular (Fig. 4d), and their GCD profiles remained symmetric and triangular (Fig. 4e). When the FSSC was charged/discharged between 0 and $1.2 \mathrm{~V}$ at $2 \mathrm{mV} \mathrm{s}^{-1}$, the areal energy and power densities were $539.4 \mu \mathrm{Wh} \mathrm{cm} \mathrm{cm}^{-2}$ and $3.24 \mathrm{~mW} \mathrm{~cm}^{-2}$, respectively. At $50 \mathrm{mV} \mathrm{s}^{-1}$, over the potential window of 0 to $1.2 \mathrm{~V}$, the energy density was $165 \mu \mathrm{Wh} \mathrm{cm}^{-2}$ and the power density was as high as $24.75 \mathrm{~mW} \mathrm{~cm}^{-2}$. As illustrated on a Ragone plot (Fig. 4f), this performance significantly exceed the current state-of-the-art ${ }^{9-19,24-26,49-51}$. For example, a FSSC formed from cellulose nanofiber-graphene aerogel electrodes provided only $18.1 \mu \mathrm{Wh} \mathrm{cm} \mathrm{cm}^{-2}$ at power density of $19.9 \mathrm{~mW}$ $\mathrm{cm}^{-226}$ and a FSSC formed using $85.6-\mu \mathrm{m}$-thick reduced graphene oxide/polypyrrole nanotube paper electrodes provided $61.4 \mu \mathrm{Wh}$ $\mathrm{cm}^{-2}$ at power density of $10 \mathrm{~mW} \mathrm{~cm}^{-2} 25$.

\section{Discussion}

A bottom-up infilling method is demonstrated to be highly effective for infilling of solid gel electrolytes into porous electrodes. This approach turns the commonly problematic skin formation and volume shrinkage issue during the gel formation into an advantage rather than a disadvantage. Because the bottom-up infilled gel electrolyte deeply penetrates the porous electrode, the 
accessibility of ions to the internal electrode surface is maximized and the ion diffusion path length is minimized, resulting in a significantly improved electrochemical performance relative to most other thick FSSCs. Because the polymer gel mechanically interlocks the porous electrode, these thick electrodes are mechanically robust and can be rolled and repeatedly bent without a reduction in capacitance. We believe this bottom-up electrode infilling strategy will be broadly useful for loading gel electrolytes into different porous electrodes because of the nearuniversality of the volume shrinkage of a gel electrolyte during solvent removal. We note that perhaps areal capacitances even higher than the present best value $\left(2662 \mathrm{mF} \mathrm{cm}^{-2}\right)$ may be possible using advanced porous pseudocapacitor electrodes ${ }^{29-31,54-56}$ if they can be formed into thick structures.

\section{Methods}

Gelation observations. $0.4 \mathrm{~mL}$ of $\mathrm{PVA} / \mathrm{H}_{3} \mathrm{PO}_{4}$ electrolyte solution is dropped onto the hydrophobic silicon wafer at room temperature for the observation of the gelation process. The $\mathrm{PVA} / \mathrm{H}_{3} \mathrm{PO}_{4}$ electrolyte solution was prepared as follows. $1 \mathrm{~g}$ of PVA ( $\mathrm{Mw} \sim 95,000 \mathrm{~g} \mathrm{~mol}^{-1}, 95 \%$ hydrolyzed, Acros) is dissolved in $15 \mathrm{~mL}$ of deionized water at $90^{\circ} \mathrm{C}$ with vigorous stirring until the solution became transparent. After cooling to room temperature, $0.8 \mathrm{~g}$ of $\mathrm{H}_{3} \mathrm{PO}_{4}$ (85 wt.\% aqueous solution, Aldrich) is added into the solution and stirred for $12 \mathrm{~h}$ at room temperature forming a homogeneous solution. For a better observation of the electrolyte solution droplet, the silicon wafer was treated in a $1 \mathrm{wt} \%$ toluene solution of heptadecafluorodecyltrimethoxysilane (FAS) for $1 \mathrm{~h}$ to make the surface hydrophobic to ensure a proper height (i.e., a larger contact angle) of the drop of electrolyte solution.

Fabrication of FSSCs. (1) Fabricating MWCNT electrodes by the bottom-up method: $9 \%$ wt. MWCNT solution $\left(9-10 \mathrm{mg} \mathrm{mL}^{-1}\right.$, Aladdin) is casted onto a horizontally placed glass substrate and dried at room temperature for $12 \mathrm{~h}$ to form a MWCNT film, whose thickness depends on the casted amount of MWCNT solution. Then the MWCNT film on the glass substrate is baked at $150^{\circ} \mathrm{C}$ for $2 \mathrm{~h}$ to reduce the electrical resistance. Then the MWCNT film is removed from the substrate by a stream of deionized water. Next, the free-standing MWCNT film is placed onto a polyvinylidene fluoride (PVDF) millipore membrane $(0.1 \mu \mathrm{m}$ pore size, Fisher Scientific), which is located on a loose sponge. A sufficient amount $(0.8$ $\mu \mathrm{L} \mathrm{cm}{ }^{-2} \mu \mathrm{m}^{-1}$ ) of $\mathrm{H}_{3} \mathrm{PO}_{4} / \mathrm{PVA} / \mathrm{H}_{2} \mathrm{O}$ electrolyte solution to fill the MWCNT film is then cast onto the film. Next, a $60 \mu \mathrm{m}$ thick PET film is placed on top of the electrolyte solution. After $24 \mathrm{~h}$ at room temperature, the sol electrolyte gelled and a free-standing gel electrolyte-infilled MWCNT, and adhered by a layer of gel electrolyte, is obtained by removing the PVDF substrate and the PET cover. The free-standing gel electrolyte infilled MWCNT electrodes are further dried at $60{ }^{\circ} \mathrm{C}$ for $12 \mathrm{~h}$.

(2) Fabricating MWCNT electrodes by the top-down method: the MWCNT solution is drop cast onto a glass substrate, dried at room temperature for $12 \mathrm{~h}$, and then baked at $150{ }^{\circ} \mathrm{C}$ for $2 \mathrm{~h}$. The same volume of electrolyte solution as for the bottom-up infilled electrode, $0.8 \mu \mathrm{L} \mathrm{cm}^{-2} \mu \mathrm{m}^{-1}$, is drop cast onto the MWCNT electrode. After $24 \mathrm{~h}$ at room temperature, the sol electrolyte gels and a freestanding MWCNT, containing the gel electrolyte in the pores and adhered by a layer of gel electrolyte, was obtained after separation from the glass substrate. Then the free-standing MWCNT electrode is further dried at $60^{\circ} \mathrm{C}$ for $12 \mathrm{~h}$.

(3) FSSC device assembly: The prepared electrodes are cut into small pieces with areas of $1 \mathrm{~cm}^{2}$. A small amount of electrolyte solution is bladed onto the gel surface of the as-prepared MWCNT electrodes, serving as a glue. Another electrode is placed face-to-face onto the electrolyte solution layer. Then a mechanical force (10 $\mathrm{kg} \mathrm{cm}^{-2}$ ) is applied to press the electrodes pair firmly together to ensure good contact between the gel layer. Finally, Cu tapes are adhered to the bare MWCNT electrode surfaces, serving as current collectors for the electrochemical testing.

(4) Fabrication of PEDOT/PSS-MWCNT-based FSSCs: A slurry of PEDOT/PSS and MWCNT is prepared as follow: $30 \mathrm{~mL}$ PEDOT/PSS aqueous solution (1.3 wt. $\%$, Aldrich) is mixed with MWCNT aqueous solution ( 9 wt.\%, Aldrich) by a volume ratio depending on the different concentration of PEDOT/PSS in the electrodes. The mixtures were vigorous stirred under $60^{\circ} \mathrm{C}$ for $3 \mathrm{~h}$, partially evaporating the water in the mixture to form viscous slurry. The viscous slurry is painted onto a PVDF millipore membrane, dried under room temperature for $12 \mathrm{~h}$, and then baked at $120^{\circ} \mathrm{C}$ for $20 \mathrm{~min}$ to form a PEDOT/PSS-MWCNT electrode. The PEDOT/PSS-MWCNT electrodes are infilled with the gel electrolyte by bottom-up (or top-down) processes and assembled into FSSC cells as described above in 'FSSC device assembly' section.

Characterization and measurements. The infilling of gel electrolyte in the pores of the MWCNT electrodes is investigated by means of field emission scanning electron microscopy (SEM, Hitachi, S-4700), energy dispersive spectroscopy (Hitachi, S-4700) and BET (NOVA 2200e). Electrochemical characterization, including CV, GCD, and EIS, is performed using a VMP3 multichannel potentiostat (VMP3, Bio-Logic, USA) and a potentiostat (Versastat 3, Princeton Applied Research, USA) in the two-electrode mode at room temperature. Contact electrodes, i.e., $\mathrm{Cu}$ tapes, are adhered to the FSSC devices for an easy connection to the probe and convenient tandems. The thicknesses of the MWCNT electrodes were measured using the cross-sectional SEM and a micrometer. Calculations of specific capacitance and energy and power densities are discussed in detail in Supplementary Note 1.

Data availability. Data used in this study is available from the corresponding authors upon request.

Received: 19 January 2017 Accepted: 17 May 2018

Published online: 03 July 2018

\section{References}

1. Lu, X., Yu, M., Wang, G., Tong, Y. \& Li, Y. Flexible solid-state supercapacitors: design, fabrication and applications. Energy Environ. Sci. 7, 2160-2181 (2014).

2. Simon, P. \& Gogotsi, Y. Materials for electrochemical capacitors. Nat. Mater. 7, 845-854 (2008).

3. Wang, X. et al. Flexible energy-storage devices: design consideration and recent progress. Adv. Mater. 26, 4763-4782 (2014).

4. Wen, L., Li, F. \& Cheng, H. M. Carbon nanotubes and graphene for flexible electrochemical energy storage: from materials to devices. Adv. Mater. 28, 4306-4337 (2016).

5. Gate, B. D. Flexible electronics. Science 323, 1566-1567 (2009).

6. Kim, D.-H. et al. Ultrathin silicon circuits with strain-isolation layers and mesh layouts for high-performance electronics on fabric, vinyl, leather, and paper. Adv. Mater. 21, 3703-3707 (2009).

7. Bauer, S. Flexible electronics: sophisticated skin. Nat. Mater. 12, 871-872 (2013).

8. Wang, X. et al. ZnS Nanostructures: synthesis, properties, and applications. Crit. Rev. Solid Stat. 38, 57-90 (2013)

9. Kim, S.-K. et al. Extremely durable, flexible supercapacitors with greatly improved performance at high temperatures. ACS Nano 9, 8569-8577 (2015).

10. Yoo, J. et al. Ultrathin planar graphene supercapacitors. Nano Lett. 11, 1423-1427 (2011)

11. Meng, F. \& Ding, Y. Sub-micrometer-thick all-solid-state supercapacitors with high power and energy densities. Adv. Mater. 23, 4098-4102 (2011).

12. Niu, Z. et al. All-solid-state flexible ultrathin micro-supercapacitors based on graphene. Adv. Mater. 25, 4035-4042 (2013).

13. El-Kady, M. F. et al. Laser scribing of high-performance and flexible graphenebased electrochemical capacitors. Science 335, 1326-1330 (2012).

14. Yun, J., Kim, D., Lee, G. \& Ha, J. S. All-solid-state flexible microsupercapacitor arrays with patterned graphene/MWNT electrodes. Carbon 79, 156-64 (2014).

15. Acerce, M., Voiry, D. \& Chhowalla, M. Metallic $1 \mathrm{~T}$ phase $\mathrm{MoS}_{2}$ nanosheets as supercapacitor electrode materials. Nat. Nanotechnol. 10, 313-318 (2015).

16. Liu, L. et al. Wearable energy-dense and power-dense supercapacitor yarns enabled by scalable graphene-metallic textile composite electrodes. Nat. Commun. 6, 7260 (2015).

17. Gao, W. et al. Direct laser writing of micro-supercapacitors on hydrated graphite oxide films. Nat. Nanotechnol. 6, 496-500 (2011).

18. Pech, D. et al. Ultrahigh-power micrometre-sized supercapacitors based on onion-like carbon. Nat. Nanotechnol. 5, 651-654 (2010).

19. Kaempgen, M. et al. Printable thin film supercapacitors using single-walled carbon nanotubes. Nano Lett. 9, 1872-1876 (2009).

20. Qi, D., Liu, Y., Liu, Z., Zhang, L. \& Chen, X. Design of architectures and materials in in-plane microsupercapacitors: current status and future challenges. Adv. Mater. 29, 1602802 (2017).

21. Wu, Z., Feng, X. \& Cheng, H. M. Recent advances in graphene-based planar micro-supercapacitors for on-chip energy storage. Nat. Sci. Rev. 1, 277-292 (2014).

22. Shen, C. et al. A Review of on-chip micro supercapacitors for integrated selfpowering systems. J. Micro. Syst. 26, 949-965 (2017).

23. Kyeremateng, N., Brousse, T. \& Pech, D. Microsupercapacitors as miniaturized energy storage components for on-chip electronics. Nat. Nanotechol. 12, 7-15 (2017)

24. $\mathrm{Xu}, \mathrm{Y}$. et al. Flexible solid-state supercapacitors based on three-dimensional graphene hydrogel films. ACS Nano 7, 4042-4049 (2013).

25. Yang, C. et al. Reduced graphene oxide/polypyrrole nanotube papers for flexible all-solid-state supercapacitors with excellent rate capability and high energy density. J. Power Sources 302, 39-45 (2016).

26. Gao, K. et al. Cellulose nanofiber-graphene all solid-state flexible supercapacitors. J. Mater. Chem. A 1, 63-63 (2013). 
27. Song, S. et al. Facile self-templating large scale preparation of biomass-derived 3D hierarchical porous carbon for advanced supercapacitors. J. Mater. Chem. A 3, 18154-18162 (2015).

28. Zhang, D. et al. Scalable synthesis of hierarchical macropore-rich activated carbon microspheres assembled by carbon nanoparticles for high rate performance supercapacitors. J. Power Sources 342, 363-370 (2017).

29. Eustache, E., Douard, C., Retoux, R., Lethien, C. \& Brousse, T. $\mathrm{MnO}_{2}$ thin films on 3D scaffold: microsupercapacitor electrodes competing with "bulk" carbon electrodes. Adv. Energy Mater. 5, 1500680 (2015).

30. El-Kady, M. et al. Engineering three-dimensional hybrid supercapacitors and microsupercapacitors for high-performance integrated energy storage. Proc. Natl Acad. Sci. USA 112, 4233-4238 (2015).

31. Ferris, A., Garbarino, S., Guay, D. \& Pech, D. $3 \mathrm{D} \mathrm{RuO}_{2}$ microsupercapacitors with remarkable areal energy. Adv. Mater. 27, 6625-6629 (2015).

32. Chen, Q. et al. Effect of different gel electrolytes on graphene-based solid-state supercapacitors. RSC Adv. 4, 36253-36256 (2014).

33. Vo, T. Q., Barisik, M. \& Kim, B. H. Near-surface viscosity effects on capillary rise of water in nanotubes. Phys. Rev. E 92, 053009 (2015).

34. Chen, X. et al. High-performance piezoelectric nanogenerators with imprinted $\mathrm{P}(\mathrm{VDF}-\mathrm{TrFE}) / \mathrm{BaTiO} 3$ nanocomposite micropillars for self-powered flexible sensors. Small 13, 1604245 (2017).

35. Cheng, Y., Lu, S., Zhang, H., Varanasi, C. \& Liu, J. Synergistic effects from graphene and carbon nanotubes enable flexible and robust electrodes for highperformance supercapacitors. Nano Lett. 12, 4206-4211 (2012).

36. Jin, Y., Chen, H., Chen, M., Liu, N. \& Li, Q. Graphene-patched CNT/MnO nanocomposite papers for the electrode of high-performance flexible asymmetric supercapacitors. ACS Appl. Mater. Interfaces 5, 3408-3416 (2013).

37. Lu, X. et al. Polypyrrole/carbon nanotube nanocomposite enhanced the electrochemical capacitance of flexible graphene film for supercapacitors. $J$. Power Sources 197, 319-324 (2012).

38. Jung, H. et al. Transparent, flexible supercapacitors from nano-engineered carbon films. Sci. Rep. 2, 773 (2012).

39. Stoller, M. \& Ruoff, R. Best practice methods for determining an electrode material's performance for ultracapacitors. Energy Environ. Sci. 3, 1294-1301 (2010).

40. Chmiola, J. et al. Monolithic carbide-derived carbon films for microsupercapacitors. Science 328, 480-483 (2010).

41. Wu, Z., Parvez, K., Feng, X. \& Müllen, K. Graphene-based in-plane microsupercapacitors with high power and energy densities. Nat. Commun. 4, 2487 (2013).

42. Kang, Y., Yoo, Y. \& Kim, W. 3-V Solid-state flexible supercapacitors with ionic-liquid-based polymer gel electrolyte for AC line filtering. ACS Appl. Mater. Interfaces 8, 13909-13917 (2016).

43. Chou, S.-L. et al. Electrodeposition of $\mathrm{MnO}_{2}$ nanowires on carbon nanotube paper as free-standing, flexible electrode for supercapacitors. Electrochem. Commun. 10, 1724-1727 (2008).

44. Kim, S.-K. et al. Selective wetting-induced micro-electrode patterning for flexible micro-supercapacitors. Adv. Mater. 26, 5108-5112 (2014).

45. Lin, $\mathrm{H}$. et al. Conducting polymer composite film incorporated with aligned carbon nanotubes for transparent, flexible and efficient supercapacitor. Sci. Rep. 3, 1353 (2013).

46. Peng, X. et al. A zwitterionic gel electrolyte for efficient solid-state supercapacitors. Nat. Commun. 7, 10600 (2016).

47. Xu, Z. et al. Electrochemical supercapacitor electrodes from sponge-like graphene nanoarchitectures with ultrahigh power density. J. Phys. Chem. Lett. 3, 2928-2933 (2012).

48. El-Kady, M. \& Kaner, R. Scalable fabrication of high-power graphene microsupercapacitors for flexible and on-chip energy storage. Nat. Commun. 4, 1475 (2013).

49. Weng, Z. et al. Graphene-cellulose paper flexible supercapacitors. Adv. Energy Mater. 1, 917-922 (2011).

50. Peng, Z. et al. Flexible and stackable laser-induced graphene supercapacitors. ACS Appl. Mater. Interfaces 7, 3414-3419 (2015).

51. Kang, Y. J. et al. All-solid-state flexible supercapacitors fabricated with bacterial nanocellulose papers, carbon nanotubes, and triblock-copolymer ion gels. ACS Nano 6, 6400-6406 (2012).
52. Snook, G., Kao, P. \& Best, A. Conducting-polymer-based supercapacitor devices and electrodes. J. Power Sources 196, 1-12 (2011).

53. Zeng, S. et al. Electrochemical fabrication of carbon nanotube/polyaniline hydrogel film for all-solid-state flexible supercapacitor with high areal capacitance. J. Mater. Chem. A 3, 23864-23870 (2015).

54. Zhi, J., Reiser, O. \& Huang, F. Hierarchical $\mathrm{MnO}_{2}$ spheres decorated by carbon-coated cobalt nanobeads: low-cost and high-performance electrode materials for supercapacitors. ACS Appl. Mater. Interfaces 8, 8452-8459 (2016).

55. Sheng, L., Jiang, L., Wei, T. \& Fan, Z. High volumetric energy density asymmetric supercapacitors based on well-balanced graphene and graphene$\mathrm{MnO}_{2}$ electrodes with densely stacked architectures. Small 12, 5217-5227 (2016).

56. Garakani, M. et al. Heterogeneous, mesoporous $\mathrm{NiCo}_{2} \mathrm{O}_{4}-\mathrm{MnO}_{2} /$ graphene foam for asymmetric supercapacitors with ultrahigh specific energies. J. Mater. Chem. A 5, 3547-3557 (2017).

\section{Acknowledgements}

Research at the University of Illinois was supported by the National Science Foundation Engineering Research Center for Power Optimization of Electro Thermal Systems (POETS) under cooperative agreement EEC-1449548. Research in China was supported by Major Research Plan of NSFC on Nanomanufacturing (Grant Number: 91323303), NSFC Funds (Grant Numbers: 51522508, 51505372), and China Postdoctoral Science Foundation (2016T90905, 2015M570824) through the State Key Laboratory for Manufacturing Systems Engineering at Xi'an Jiaotong University. We thank Mr. Wei Yu for discussion on the comment about mechanical performance of FSSCs.

\section{Author contributions}

J.S. and X.L. conceptualized the idea and designed the experiments. P.V.B. supervised the project. X.L. and C.Y. performed the infiltration experiments. X.L., S.-K.K., and J.W. tested and analyzed the electrochemical performances of FSSCs. X.L. tested mechanical performances of FSSCs. Y.M., Q.Z., P.S., and R.Z. commented and tested the porosities of electrodes. X.L. and P.V.B. co-wrote the manuscript.

\section{Additional information}

Supplementary Information accompanies this paper at https://doi.org/10.1038/s41467018-04937-8.

Competing interests: The authors declare no competing interests.

Reprints and permission information is available online at http://npg.nature.com/ reprintsandpermissions/

Publisher's note: Springer Nature remains neutral with regard to jurisdictional claims in published maps and institutional affiliations.

Open Access This article is licensed under a Creative Commons Attribution 4.0 International License, which permits use, sharing, adaptation, distribution and reproduction in any medium or format, as long as you give appropriate credit to the original author(s) and the source, provide a link to the Creative Commons license, and indicate if changes were made. The images or other third party material in this article are included in the article's Creative Commons license, unless indicated otherwise in a credit line to the material. If material is not included in the article's Creative Commons license and your intended use is not permitted by statutory regulation or exceeds the permitted use, you will need to obtain permission directly from the copyright holder. To view a copy of this license, visit http://creativecommons.org/ licenses/by/4.0/.

(c) The Author(s) 2018 